\title{
Bicoloured Dyck paths and the contact polynomial for $n$ non-intersecting paths in a half-plane lattice.
}

\author{
R. Brak† and J. W. Essam†* \\ $\dagger$ Department of Mathematics, \\ The University of Melbourne \\ Parkville, Victoria 3052, Australia \\ $\ddagger$ Department of Mathematics, \\ Royal Holloway College, University of London, \\ Egham, Surrey TW20 0EX, England.
}

Submitted: May 14, 2001; Accepted: Feb 22, 2003; Published: Sep 12, 2003

MR Subject Classifications: 05A15

\begin{abstract}
In this paper configurations of $n$ non-intersecting lattice paths which begin and end on the line $y=0$ and are excluded from the region below this line are considered. Such configurations are called Hankel $n$-paths and their contact polynomial is defined by $\hat{Z}_{2 r}^{\mathrm{H}}(n ; \kappa) \equiv \sum_{c=1}^{r+1}\left|\mathcal{H}_{2 r}^{(n)}(c)\right| \kappa^{c}$ where $\mathcal{H}_{2 r}^{(n)}(c)$ is the set of Hankel $n$-paths which make $c$ intersections with the line $y=0$ the lowest of which has length $2 r$. These configurations may also be described as parallel Dyck paths.

It is found that replacing $\kappa$ by the length generating function for Dyck paths, $\kappa(\omega) \equiv \sum_{r=0}^{\infty} C_{r} \omega^{r}$, where $C_{r}$ is the $r^{t h}$ Catalan number, results in a remarkable simplification of the coefficients of the contact polynomial. In particular it is shown that the polynomial for configurations of a single Dyck path has the expansion $\hat{Z}_{2 r}^{\mathrm{H}}(1 ; \kappa(\omega))=\sum_{b=0}^{\infty} C_{r+b} \omega^{b}$. This result is derived using a bijection between bicoloured Dyck paths and plain Dyck paths. A bi-coloured Dyck path is a Dyck path in which each edge is coloured either red or blue with the constraint that the colour can only change at a contact with the line $y=0$. For $n>1$, the coefficient of $\omega^{b}$ in $\hat{Z}_{2 r}^{\mathrm{W}}(n ; \kappa(\omega))$ is expressed as a determinant of Catalan numbers which has a combinatorial interpretation in terms of a modified class of $n$ non-intersecting Dyck paths. The determinant satisfies a recurrence relation which leads to the proof of a product form for the coefficients in the $\omega$ expansion of the contact polynomial.
\end{abstract}

*email: r.brak@ms.unimelb.edu.au, j.essam@alpha1.rhbnc.ac.uk 


\section{Introduction.}

We consider $n$ non-intersecting paths on the half plane square lattice $\Xi=\{(t, y) \mid t, y \in$ $\mathbb{Z}, y \geq 0$ and $t+y$ even $\}$, where $\mathbb{Z}$ is the set of integers. The results in this paper follow recent interest in single paths on $\Xi[1], n$ non-intersecting paths [2],[3] and with their applications in statistical mechanics [4],[5], as well as their connection with the Bethe Ansatz of statistical mechanics [1],[6] and work on return polynomials [7].

A single path known as a ballot path is defined as follows.

Definition 1 (Ballot and Dyck paths). A path of length $t \geq 0$ is a sequence of vertices $\left(v_{0}, v_{1}, \ldots, v_{t}\right), v_{i} \in \Xi$, with $v_{i}-v_{i-1}=(1,1)$ (an up step) or $(1,-1)$ (a down step), $i=1, \ldots, t . A t=0$ length path is a single vertex $v_{0} . A$ ballot path of length $t$ with deviation $y \geq 0$ has $v_{0}=(0,0)$ and $v_{t}=(t, y)$. Ballot paths with zero deviation are known as Dyck paths and the set of Dyck paths of length $2 r \geq 0$ will be denoted by $\{\triangle \mathcal{M}\}_{2 r}$. Denote the length of a path $\pi$ by $|\pi|$.

The number of ballot paths with deviation $y, B_{t, y}$, is called a ballot number and is given by

$$
B_{t, y}=\frac{(y+1) t !}{\left(\frac{1}{2}(t+y)+1\right) !\left(\frac{1}{2}(t-y)\right) !}
$$

The case $y=0, t=2 r$ gives the number of Dyck paths of length $2 r$

$$
\left|\{M\}_{2 r}\right|=B_{2 r, 0}=\frac{1}{r+1}\left(\begin{array}{c}
2 r \\
r
\end{array}\right)=C_{r}
$$

which is the $r^{\text {th }}$ Catalan number. The Dyck path length generating function is

$$
\kappa(\omega) \equiv \sum_{r=0}^{\infty}\left|\{\Delta\}_{2 r}\right| \omega^{r}=\sum_{r=0}^{\infty} C_{r} \omega^{r}
$$

This series can be summed to give

$$
\kappa(\omega)=\frac{1-\sqrt{1-4 \omega}}{2 \omega}
$$

which is a root of the quadratic $\kappa=1+\omega \kappa^{2}$. The " $\omega$ " variable arises naturally in certain physical applications of the contact polynomials [4], [5].

Definition 2 (Surface and Contacts). The line $y=0$ will be referred to as the surface. Any vertex of a Dyck path in common with the surface, is called a contact. Denote the

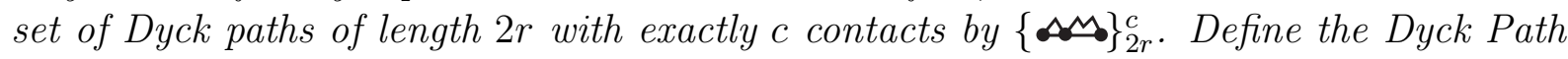
contact polynomial by

$$
\hat{Z}_{2 r}^{\mathcal{S}}(\kappa) \equiv \sum_{c=1}^{r+1}\left|\{\circlearrowleft\}_{2 r}^{c}\right| \kappa^{c} .
$$


The notation $\hat{Z}^{\mathcal{S}}$ agrees with that used in our earlier work [1] where the contact polynomial for paths with only one end attached to the surface were denoted $Z^{S}$. The superscript $S$ denotes a single path.

A somewhat surprising result is obtained if, starting with any Dyck path contact polynomial, the variable $\kappa$ is replaced by the Dyck path length generating function $\kappa(\omega)$. Thus with the definition

$$
\hat{H}_{2 r}^{\mathcal{S}}(\omega) \equiv \hat{Z}_{2 r}^{\mathcal{S}}(\kappa(\omega))
$$

we find, for example

$$
\begin{aligned}
\hat{H}_{4}^{\mathcal{S}}(\omega) & =\kappa(\omega)^{2}+\kappa(\omega)^{3} \\
& =\left(1+\omega+2 \omega^{2}+5 \omega^{3}+14 \omega^{4}+\ldots\right)^{2}+\left(1+\omega+2 \omega^{2}+5 \omega^{3}+14 \omega^{4}+\ldots\right)^{3} \\
& =2+5 \omega+14 \omega^{2}+42 \omega^{3}+\ldots=\sum_{b=0}^{\infty} C_{b+2} \omega^{b}
\end{aligned}
$$

which is a case of the first result of this paper

$$
\hat{H}_{2 r}^{\mathcal{S}}(\omega)=\sum_{b=0}^{\infty} C_{r+b} \omega^{b} .
$$

Notice that (3) is the case $r=0$ of (10) since $\hat{Z}_{0}^{\mathcal{S}}(\kappa(\omega))=\kappa(\omega)$. On the other hand it follows from (4) that setting $\omega=0$ in (10) is equivalent to setting $\kappa=1$ which gives

$$
\hat{Z}_{2 r}^{\mathcal{S}}(1)=C_{r}
$$

being the total number of Dyck paths of length $2 r$.

Equation (10) has already been proved analytically in [1], we now provide a combinatorial proof. A combinatorial interpretation of $\hat{Z}_{2 r}^{\mathcal{S}}(\kappa(\omega))$ can be obtained as follows. The substitution and expansion of $\kappa(\omega)$ gives rise to a series of terms each one of which can be made to correspond to a Dyck path in which the steps are assigned one of two colours as follows. Each member of a subset of the contacts of the original Dyck path is replaced by another Dyck path of length at least two. Contacts in the complementary set are unchanged corresponding to choosing the first term, $C_{0}=1$, in the expansion of $\kappa(\omega)$. To distinguish the steps of the original path from the steps of the inserted paths we will colour the former red and the latter blue. This leads to the idea of bi-coloured Dyck paths.

Definition 3 (Bi-coloured Dyck path). A bi-coloured Dyck path, $D_{r, b}$, is a Dyck path in which each edge is coloured either red or blue with the constraint that the colour can only change at a contact. Denote the set of bi-coloured Dyck paths having $2 r$ red steps and $2 b$ blue steps by $\{\boldsymbol{\sim}\}_{2 r, 2 b}$.

Examples of bi-coloured paths are illustrated in figure 1. 


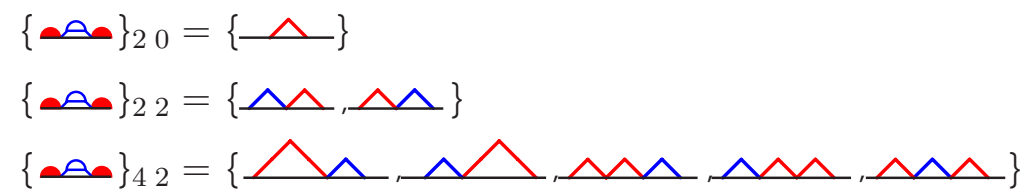

Figure 1: An example of the first few sets of bi-coloured Dyck paths.

Since a factor $\omega$ arises from each step of the inserted (blue) paths we have that

$$
\hat{Z}_{2 r}^{\mathcal{S}}(\kappa(\omega))=\sum_{b=0}^{\infty}\left|\{\boldsymbol{\sim}\}_{2 r, 2 b}\right| \omega^{b} .
$$

In section 2.2 we prove that equation (10) is equivalent to (12) and the following theorem.

Theorem 1. The number of bi-coloured Dyck paths with $2 r$ red steps and $2 b$ blue steps is given by

$$
\left|\{\boldsymbol{\sim}\}_{2 r, 2 b}\right|=C_{r+b} .
$$

The result that $\left|\{\boldsymbol{\sim}\}_{2 r, 2 b}\right|$ depends only on $r+b$ is consistent with the symmetry between red and blue in the definition.

In section 2 we will provide a combinatorial proof of theorem 1 using a bijection between $\{\boldsymbol{\sim}\}_{2 r, 2 b}$ and plain Dyck paths of length $2 r+2 b$. As an example, for the paths in figure 1 , we have bijections between the sets illustrated in figure 2 .

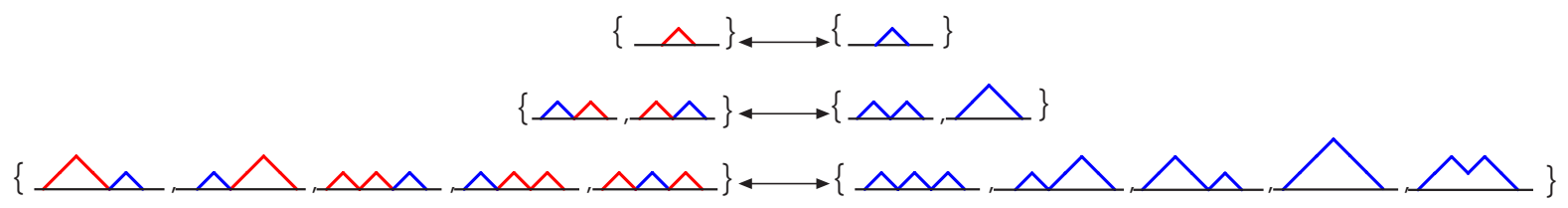

Figure 2: Example of the sets the bi-coloured paths which are in bijection with those of figure 1. Note, $r$ and $b$ are fixed in each row.

The bijection uses the marking of one step of each plain Dyck path as the bridge connecting a ballot path and a reversed ballot path. The resulting marked Dyck path will be known as a bi-ballot path and is defined as follows.

Definition 4 (Bi-ballot paths). A bi-ballot path with parameters $r>0$ and $b \geq 0$ is a Dyck path of length $2 r+2 b>0$ having a marked up edge, known as its bridge (marked by $a \sharp$ ), which is the step immediately before the path intersects the bi-ballot line $\mathcal{L}$ of slope -1 through the point $(2 r, 0)$ (see figure 3). Denote the set of such paths by $\{\stackrel{M}{\Delta}\}_{2 r, 2 b}$. The height $m$ of a bi-ballot path is the $y$-coordinate of the terminal vertex of its bridge, thus the bridge connects vertices $(2 r-m-1, m-1)$ and $(2 r-m, m)$. Denote the set of bi-ballot paths of given height by $\left\{\stackrel{M^{*}}{\Delta}\right\}_{2 r, 2 b}^{m}$. 


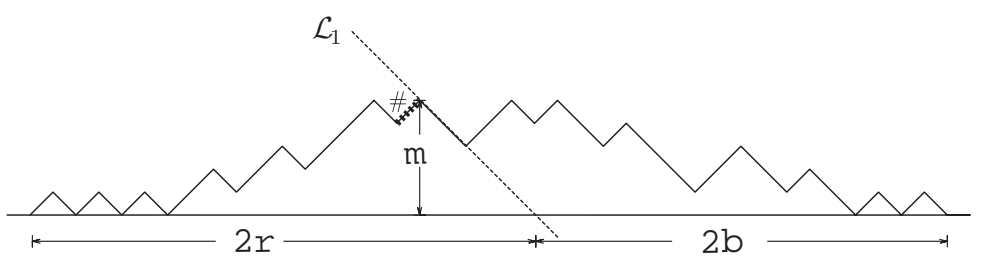

Figure 3: An example of a fixed bi-ballot path of height $m$.

We will obtain a bijection between $\{\stackrel{M}{M}\}_{2 r, 2 b}$ and $\{\boldsymbol{n}\}_{2 r, 2 b}$ (see lemma 3 ) and hence from (12)

$$
\hat{Z}_{2 r}^{\mathcal{S}}(\kappa(\omega))=\sum_{b=0}^{\infty}\left|\{\stackrel{4}{\Delta}\}_{2 r, 2 b}\right| \omega^{b}
$$

We now partition the set of all bi-ballot paths with given $r$ and $b$ by height and since this set has the same cardinality as the set of plain Dyck paths of length $2 r+2 b$ it follows that

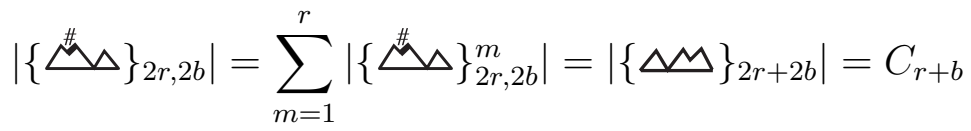

and the theorem is proved once the bijection is shown.

Remark 1. A bi-ballot path of height $m$ is the concatenation of a ballot path of length $2 r-m-1$ and height $m-1$ with its bridge (which is always up) and a reversed ballot path of length $2 b+m$ and height $m$ so

$$
\left|\{\stackrel{\text { 共 }}{\text { 作 }}\}_{2 r, 2 b}^{m}\right|=B_{2 r-m-1, m-1} B_{2 b+m, m}
$$

and using (15)

$$
\sum_{m=1}^{r} B_{2 r-m-1, m-1} B_{2 b+m, m}=C_{r+b}
$$

or

$$
\sum_{m=1}^{r} \frac{m}{r}\left(\begin{array}{c}
2 r-m-1 \\
r-1
\end{array}\right) \frac{m+1}{b+m+1}\left(\begin{array}{c}
2 b+m \\
b
\end{array}\right)=\frac{1}{r+b+1}\left(\begin{array}{c}
2 r+2 b \\
r+b
\end{array}\right)
$$

which is the analogue of the Chu-Vandemonde summation formula for Dyck paths. Similar results may be found in Gould [8].

In section 3 equation (10) is extended to the case of Hankel $n$-path configurations. 
Definition 5 (Hankel $n$-path). A Hankeln-path is a configuration of n non-intersecting paths (ie. no vertices in common between any pair of paths) on $\Xi$ indexed by $\alpha=1, \ldots, n$ in which path $\alpha$ begins at vertex $(-2(\alpha-1), 0)$ and ends at vertex $(2(r+\alpha-1), 0)$. The shortest of these paths has length $2 r$. The set of such configurations having c contacts will be denoted by $\mathcal{H}_{2 r}^{(n)}(c)$ and the watermelon contact polynomial is defined as

$$
\hat{Z}_{2 r}^{H}(n ; \kappa)=\sum_{c=2 n-1}^{2 n+r-1}\left|\mathcal{H}_{2 r}^{(n)}(c)\right| \kappa^{c}
$$

We use this name since the total number of such configurations is enumerated by a Hankel determinant [10] (see for example equation (32) below). The individual paths of a Hankel $n$-path are clearly Dyck paths. An example of a Hankel 3-path is shown in figure 4a.

a)

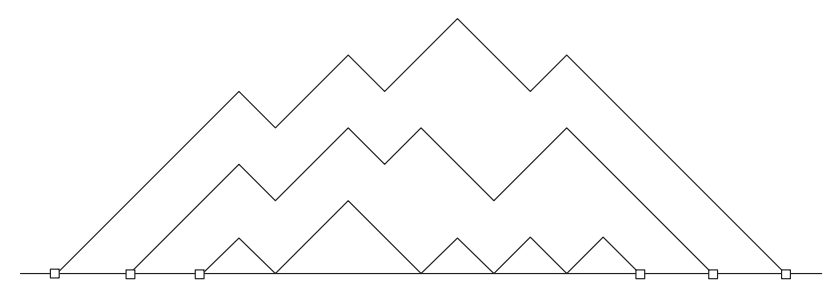

b)

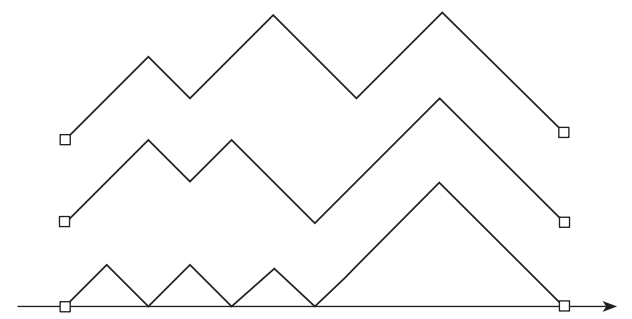

Figure 4: a) An example of a Hankel 3-path. b) An example of three path watermelon configuration.

We now consider the analogous operation used in formulating (10), ie. replacing $\kappa$ by $\kappa(\omega)$, in the case of Hankel paths and question if this gives anything interesting. Since $\hat{Z}_{2 r}^{\mathrm{H}}(n ; \kappa)$ is a contact polynomial, all the coefficients of $\kappa$ are necessarily positive integers. Furthermore, the coefficients of $\omega$ in $\kappa(\omega)$ are likewise positive and hence the substitution $\kappa \rightarrow \kappa(\omega)$ will obviously produce a series in $\omega$ whose coefficients are positive. We now ask, "What do these coefficients enumerate"? A direct application of bi-coloured path interpretation does not lead to anything interesting, since, if the steps of the Hankel path are coloured red, and the Dyck paths that are inserted in place of the contacts of the Hankel paths are coloured blue, the resulting path configurations will, in most cases, lead to a set of intersecting (bi-coloured) paths. Is there a different interpretation in which the coefficients enumerate non-intersecting configurations of some type of path? The answer turns out to be "Yes". The non-intersecting configurations we will obtain are a modified class of Hankel paths where the uppermost path ends a distance $2 b$ further along the $t$-axis - see figure 5. We will call such path configurations "Ceratic" Hankel paths ${ }^{1}$.

$\hat{H}_{2 r}^{(n)}(\omega)$ is defined by replacing $\kappa$ by $\kappa(\omega)$ in $\hat{Z}_{2 r}^{\mathrm{H}}(n ; \kappa)$ and expanding in powers of $\omega$

\footnotetext{
${ }^{1}$ The paths are called thus because of the similarity to the Abyssal Anglerfish, Ceratias hollbolli.
} 


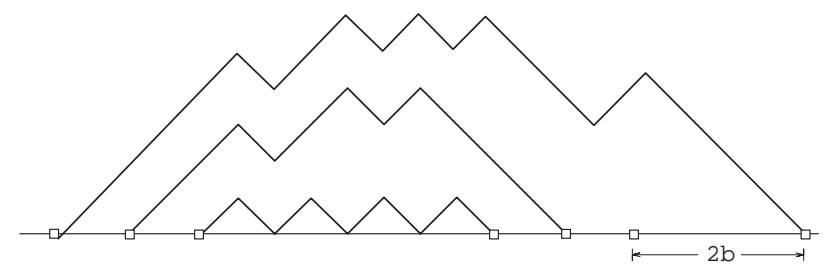

Figure 5: The combinatorial interpretation of the coefficients of the $\omega$ expansion - the uppermost path terminates a distance $2 b$ further along the axis. We call these paths "Ceratic" Hankel paths with parameter $b$.

defines $\hat{h}_{r}^{(n)}(b)$ as the coefficient of $\omega^{b}$.

$$
\hat{H}_{2 r}^{(n)}(\omega) \equiv \hat{Z}_{2 r}^{\mathrm{H}}(n, \kappa(\omega))=\sum_{b=0}^{\infty} \hat{h}_{r}^{(n)}(b) \omega^{b}
$$

The main result of this paper is theorem 3 which shows that $\hat{h}_{r}^{(n)}(b)$ can be written as a determinant of Catalan numbers; see (43). The theorem is proved by applying a recurrence relation for the single path function, $\hat{H}_{2 r}^{\mathcal{S}}(\omega)$, to the elements of the Gessel-Viennot determinant and also independently by a variation of the Gessel-Viennot method. The interpretation of $\omega$ as the number of Ceratic Hankel paths follows from the determinant using the Gessel-Viennot theorem (theorem 2). Furthermore, theorem 4, the determinant may be evaluated to express $\hat{h}_{r}^{(n)}(b)$ in the product form

$$
\hat{h}_{r}^{(n)}(b)=\left(\begin{array}{c}
n+b-1 \\
b
\end{array}\right) \frac{(2 r+2 n-1)_{2 b}}{(r+n)_{b}(r+2 n)_{b}} \prod_{i=1}^{n} \frac{(2 r+2 i-2) !(2 i-1) !}{(r+i-1) !(r+i+n-1) !}
$$

where $(a)_{k}$ denotes the rising factorial $a(a+1) \ldots(a+k-1)$. We also show that $\hat{H}_{2 r}^{(n)}(\omega)$ satisfies the recurrence relations (50), (52) and (56).

In polymer physics applications [9] the $n$ path configurations usually considered are known as "Watermelons".

Definition 6 (Watermelon). A watermelon ${ }^{2}$ with zero deviation and length $2 r$ is a configuration of $n$ non-intersecting paths on $\Xi$ indexed by $\alpha=1, \ldots n$ in which path $\alpha$ begins at vertex $(0,2(\alpha-1))$ and ends at vertex $(2 r, 2(\alpha-1))$. The set of such configurations for which the path for $\alpha=1$ has c contacts will be denoted by $\mathcal{W}_{2 r}^{(n)}(c)$ and the watermelon contact polynomial is defined as

$$
\hat{Z}_{2 r}^{W}(n ; \kappa)=\sum_{c=1}^{r+1}\left|\mathcal{W}_{2 r}^{(n)}(c)\right| \kappa^{c}
$$

\footnotetext{
${ }^{2}$ This name was used by Fisher[9] presumably because of the similarity to the stripes on a watermelon.
} 
Note: $\left|\mathcal{W}_{2 r}^{(n)}(1)\right|=\delta_{r, 0}$

An example of a watermelon configuration is shown in figure $4 \mathrm{~b}$. There is an obvious bijection between watermelons and Hankel paths. Each watermelon corresponds to a distinct Hankel $n$-path by connecting its initial and final vertices to the $t$ - axis by the shortest possible non-intersecting paths (see figure 4). The contact polynomial $\hat{Z}_{2 r}^{\mathrm{H}}(n ; \kappa)$ for Hankel $n$-paths is related to that for watermelons by

$$
\hat{Z}_{2 r}^{\mathrm{H}}(n ; \kappa)=\kappa^{2(n-1)} \hat{Z}_{2 r}^{\mathrm{W}}(n ; \kappa)
$$

since for Hankel configurations all $n$ paths have both ends in contact with the surface. Both polynomials reduce to the contact polynomial for a single Dyck path in the case $n=1$.

The following recurrence relations for $\hat{Z}_{2 r}^{(n)}(\kappa) \equiv \hat{Z}_{2 r}^{\mathrm{W}}(n ; \kappa)$ may be shown to be equivalent to (50), (52) and (56) for Hankel $n$-paths by using (23), (20) and $\omega=(\kappa-1) / \kappa^{2}$, which follows from (4).

$$
\begin{aligned}
(\kappa-1) \hat{Z}_{2 r-2}^{(n-1)}(1) \hat{Z}_{2 r}^{(n)}(\kappa) & =\kappa^{2} \hat{Z}_{2 r}^{(n-1)}(1) \hat{Z}_{2 r-2}^{(n)}(\kappa)-\hat{Z}_{2 r-2}^{(n)}(1) \hat{Z}_{2 r}^{(n-1)}(\kappa) \\
\kappa^{2} \hat{Z}_{2 r+4}^{(n-2)}(1) \hat{Z}_{2 r}^{(n)}(\kappa) & =\hat{Z}_{2 r}^{(n-1)}(1) \hat{Z}_{2 r+4}^{(n-1)}(\kappa)-\hat{Z}_{2 r+2}^{(n-1)}(1) \hat{Z}_{2 r+2}^{(n-1)}(\kappa)
\end{aligned}
$$

and, of particular significance,

$$
\begin{aligned}
(r+2 n-2)(r+n-1)_{n-1}^{2}(\kappa-1) \hat{Z}_{2 r}^{(n)}(\kappa) & \\
-4^{n-1}\left(r-\frac{1}{2}\right)_{n-1}(r+n-1)_{n-1}\{ & \left.2(2 r-3)(\kappa-1)+(r+2 n-2) \kappa^{2}\right\} \hat{Z}_{2 r-2}^{(n)}(\kappa) \\
& +16^{n-1} 2(2 r-3)\left(r-\frac{1}{2}\right)_{n-1}^{2} \kappa^{2} \hat{Z}_{2 r-4}^{(n)}(\kappa)=0
\end{aligned}
$$

The last of these equations was deduced from the first two in [5] and has the merit of being an ordinary difference equation, relating the polynomials for different lengths but a fixed number of paths. It was used in [5] to discuss the scaling properties of the free energy of a polymer network.

\section{Enumeration of Bi-coloured Dyck paths.}

\subsection{Catalan and Dyck factors.}

In order to obtain the required bijection between bi-coloured Dyck paths and bi-ballot paths we need the following factorizations. The bijection is then shown by rearranging the factors.

Definition 7 (Catalan Factor). The set of Catalan factors, or C-factors, of length $2 r$, $\{\triangle\}_{2 r}$, is the subset of Dyck paths defined by

$$
\{\Delta\}_{2 r}=\left\{\pi \mid \pi \in\{\leadsto\}_{2 r}^{c} \text {, with } c=2\right\}
$$


If $\pi \in\{\triangleq\}_{2 r}$ we will refer to the leftmost step as the left leg and the rightmost step as the right leg.

Clearly C-factors are just Dyck paths which only have the first and last vertices in common with the surface. These paths are also sometimes called raised or elevated Dyck paths.

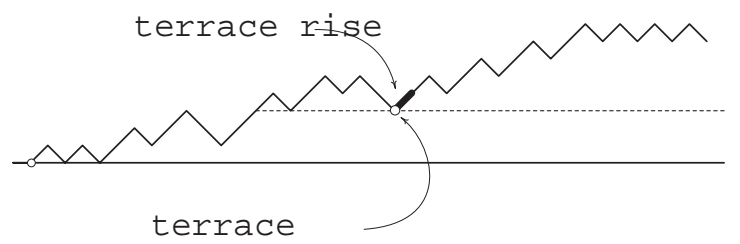

Figure 6: An example showing a height $g=3$ terrace and the corresponding terrace edge and vertex

Definition 8 (Terraces). For $g \geq 0$ the height $g$ terrace is the horizontal line $y=g$ on $\Xi$. The height $g$ terrace vertex, $v$, of a ballot path of height $h>g$ is its rightmost vertex in common with the height $g$ terrace. The height $g$ terrace rise of a ballot path is its edge having left vertex $v$. (See figure 6).

Note, since $g>h$, the terrace vertex and rise always exist.

a)

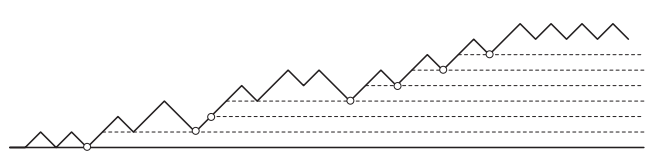

b)

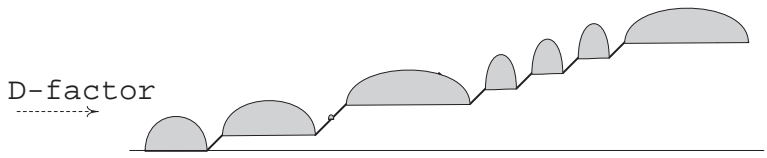

Figure 7: An example (a) of the use of seven terraces $(g=0, \ldots, 6)$ to uniquely factorise the height seven ballot path into eight D-factors (b) - one of which is a zero step path.

Definition 9 (Dyck factor). A Dyck factor or D - factor of height $\alpha$ is Dyck path of maximum length, which begins and ends on terrace $\alpha$ and never passes below it.

Notice that removing the legs from a C-factor gives a D-factor of height one.

Lemma 1. A bi-coloured Dyck path $D_{r, b}$ with $r \geq 1$ can be uniquely factored in the form

$$
D_{r, b}=\beta_{0}\left(\rho_{1} \beta_{1} \rho_{2} \beta_{2} \ldots \beta_{m-1} \rho_{m} \beta_{m}\right) \quad \text { for some } \quad m \in\{1,2, \ldots, r\}, \quad r \geq 1
$$

where $\beta_{\alpha} \in\{\Delta \boldsymbol{M}\}, \rho_{\alpha} \in\{\Delta\},\left|\beta_{0}\right|+\ldots+\left|\beta_{m}\right|=2 b$ and $\left|\rho_{1}\right|+\ldots+\left|\rho_{m}\right|=2 r$. All the edges in the $\beta_{\alpha}$ paths are coloured blue and those in $\rho_{\alpha}$ red. If $\beta_{\alpha}$ is the zero step path, then the single vertex is coloured blue. Denote the set of bi-coloured Dyck paths having $2 r$ red steps, $2 b$ blue steps and $m$ red Catalan factors by $\{\boldsymbol{n}\}_{2 r, 2 b}^{m}$ 
Proof. A Dyck path is factored by its internal contacts into Catalan factors. By definition of a bi-coloured Dyck path each factor must be of a single colour. The $\beta_{\alpha}$ of positive length are obtained by concatenating adjacent blue C-factors. A contact separating adjacent red C-factors is coloured blue to give a $\beta_{\alpha}$ of zero length.

The bicoloured paths are partitioned by the number of red Catalan factors so

$$
\left|\{\boldsymbol{\sim}\}_{2 r, 2 b}\right|=\sum_{m=1}^{r}\left|\{\boldsymbol{\sim}\}_{2 r, 2 b}^{m}\right| .
$$

Lemma 2. Any ballot path of length $2 r+y$ and height $y>0$ can be uniquely written in the form $\beta_{0} u_{1} \beta_{1} u_{2} \beta_{2} \ldots u_{y} \beta_{y}$ where $\beta_{\alpha} \in\{\boldsymbol{M}\},\left|\beta_{0}\right|+\ldots+\left|\beta_{y}\right|=2 r$ and $u_{\alpha}$ is the height $\alpha-1$ terrace rise.

Proof. The terrace vertices of the ballot path of heights $\alpha=0,1, \ldots y-1$ partition the path into $y+1$ subpaths. The first of these is the Dyck path $\beta_{0}$ and the others are of the form $u_{\alpha} \beta_{\alpha}, \alpha=1, \ldots y$, where $u_{\alpha}$ is a terrace rise and $\beta_{\alpha}$ is a D-factor of height $\alpha$.

\subsection{The number of bi-coloured Dyck paths.}

Lemma 3. There exists a bijection, $\Gamma:\{\boldsymbol{\Omega}\}_{2 r, 2 b}^{m} \stackrel{\text { biject }}{\longleftrightarrow}\left\{\stackrel{M^{*}}{\Delta}\right\}_{2 r, 2 b}^{m}$, between the set of bi-coloured Dyck paths of length $2 r$ having $m$ red Catalan factors and bi-ballot paths with bi-ballot line through $(2 r, 0)$, having bridge height $m$, and hence

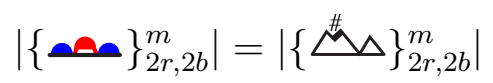

a)

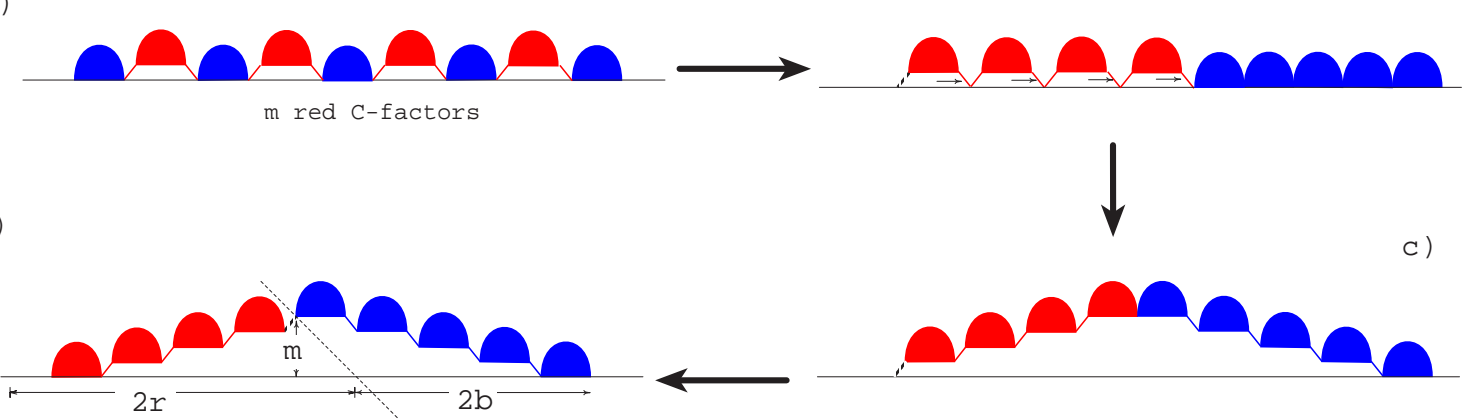

Figure 8: Several stages of bi-coloured path bijection. 
Proof. We first define the mapping $\Gamma$, and then show that it is a bijection. Let $\pi=D_{r, b} \in$ $\{\boldsymbol{\sim}\}_{2 r, 2 b}^{m}$ be written in the form (27) (see figure 8a). First, shift all the blue edges (or contacts) to the right to form the path (see figure $8 \mathrm{~b}$ )

$$
\pi \rightarrow \pi^{\prime}=\rho_{1} \rho_{2} \ldots \rho_{m} \beta_{0} \beta_{1} \ldots \beta_{m}
$$

then take the right leg of each $\rho_{\alpha}$ and "intercolate" it between the corresponding pair of blue paths (see figure 8c) i.e. if $\rho_{\alpha}=u_{\alpha} \hat{\rho}_{\alpha} \bar{u}_{\alpha}$ then

$$
\pi^{\prime} \rightarrow \pi^{\prime \prime}=u_{1} \hat{\rho}_{1} u_{2} \hat{\rho}_{2} \ldots u_{m} \hat{\rho}_{m} \beta_{0} \bar{u}_{1} \beta_{1} \bar{u}_{2} \beta_{2} \ldots \bar{u}_{m} \beta_{m} \quad \text { (Intercolate red right legs) }
$$

Finally, "left-shuffle" the red path by deleting $u_{1}$ and inserting it between $\hat{\rho}_{m} \beta_{0}$ of $\pi^{\prime \prime}$ (see figure $8 \mathrm{~d}$ ), to give

$$
\pi^{\prime \prime} \rightarrow \pi^{\prime \prime \prime}=\hat{\rho}_{1} u_{2} \hat{\rho}_{2} \ldots u_{m} \hat{\rho}_{m} u_{1} \beta_{0} \bar{u}_{1} \beta_{1} \bar{u}_{2} \beta_{2} \ldots \bar{u}_{m} \beta_{m} \quad \text { (Left-shuffle red path) }
$$

Removing the colours from $\pi^{\prime \prime \prime}$ gives a fixed bi-ballot path in $\left\{\stackrel{A^{\prime}}{\Delta}\right\}_{2 r, 2 b}^{m}$ where $u_{1}$ is the bridge. (The bridge partitions the Dyck path into two subpaths $\hat{\rho_{1}} u_{2} \hat{\rho_{2}} u_{3} \ldots u_{m} \hat{\rho_{m}}$ and $\beta_{0} \bar{u}_{1} \beta_{1} \bar{u}_{2} \beta_{2} \ldots \bar{u}_{m} \beta_{m}$ which, by Lemma 2 , are a ballot paths (the latter written in reverse order) with deviations $m-1$ and $m$ respectively.)

The mapping $\Gamma$ is inverted as follows. Starting with $\pi^{\prime \prime \prime} \in\left\{\stackrel{M^{*}}{\Delta}\right\}_{2 r, 2 b}^{m}$, colour the path to the right of $\sharp$, blue, that to the left, red. Draw all terraces of heights $0,1, \ldots, m-1$. These terraces define the D-factors of the red and blue paths. The path can now be uniquely written in the form

$$
\bar{\pi}^{\prime \prime \prime}=\hat{\rho}_{1} u_{2} \hat{\rho}_{2} \ldots u_{m} \hat{\rho}_{m} u_{1} \beta_{0} \bar{u}_{1} \beta_{1} \bar{u}_{2} \beta_{2} \ldots \bar{u}_{m} \beta_{m}
$$

(D-factorised)

where the $\beta_{\alpha}$ and the $\hat{\rho}_{\alpha}$ are the blue and red D-factors, respectively. Now, shift the bridge edge to the extreme left end ie. "right-shuffle", and set its colour to red, to give

$$
\bar{\pi}^{\prime \prime \prime} \rightarrow \bar{\pi}^{\prime \prime}=u_{1} \hat{\rho}_{1} u_{2} \hat{\rho}_{2} \ldots u_{m} \hat{\rho}_{m} \beta_{0} \bar{u}_{1} \beta_{1} \bar{u}_{2} \beta_{2} \ldots \bar{u}_{m} \beta_{m} . \quad \text { (right-shuffled reds) }
$$

Finally, shift the blue legs, $\bar{u}_{\alpha}$ to the left and change their colour to red, ie. "left intercolate blue rises" to give

$$
\bar{\pi}^{\prime \prime} \rightarrow \bar{\pi}=\beta_{0}\left(\rho_{1} \beta_{1} \rho_{2} \beta_{2} \ldots \beta_{m} \rho_{m} \beta_{m}\right), \quad \text { (left intercolated blue rises) }
$$

where $\rho_{\alpha}=u_{\alpha} \hat{\rho}_{\alpha} \bar{u}_{\alpha}$. Clearly $\bar{\pi} \in\{\boldsymbol{\Omega}\}_{2 r, 2 b}^{m}$ and $\bar{\pi}=\pi$, and hence $\Gamma^{-1} \circ \Gamma=1$ thus $\Gamma$ is a bijection.

A particular example of the bijection is shown in figure 9 .

Proof of theorem 1. The theorem follows from equations (28), (15) and Lemma 3.

$$
=\left(t_{\alpha}^{i}, y_{\alpha}^{i}\right)=\left(t_{\alpha}^{f}, y_{\alpha}^{f}\right)
$$


a)

b)

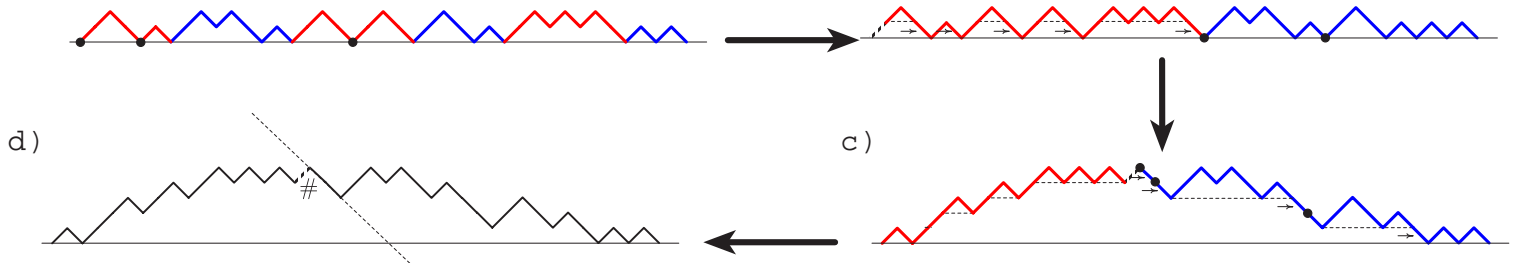

e)

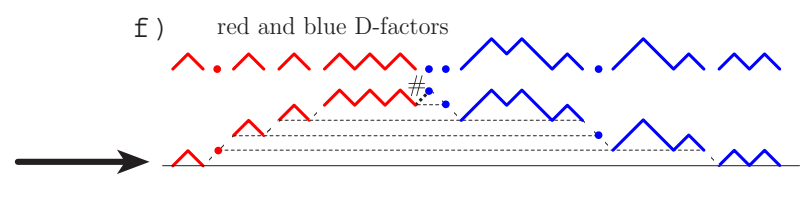

h)

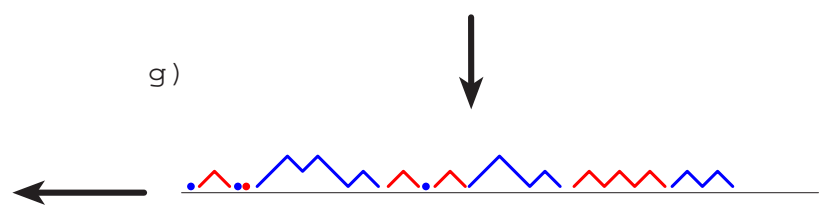

Figure 9: (a) - (d) The two-coloured path bijection applied to a particular example. (e) - (h) The bijection applied to the final path d), showing that the original path, a) is reobtained ie. that $\Gamma^{2}=1$. Note, in g) the path corresponding to $\bar{\pi}$ is illustrated without the $u$ and $\bar{u}$ steps (which are then inserted in $h$ )).

\section{The $\omega$-expansion for Hankel $n$-paths.}

\subsection{Expressing the Hankel $n$-path contact polynomial as a de- terminant.}

In the case of two or more paths we take as our starting point the following theorem

Theorem 2. (Gessel and Viennot [11, 12]) For $n>1$ let $Z\left(\mathbf{v}^{\mathbf{i}}, \mathbf{v}^{\mathbf{f}}\right)$ be a weighted sum over configurations of $n$ non-intersecting paths on a directed graph, in which path $\alpha$ starts at vertex $v_{\alpha}^{i}$ and ends at vertex $v_{\alpha}^{f}$. Suppose that the weight attached to a given path is a product of weights associated with vertices and arcs visited by the paths. If there is at least one non-intersecting configuration and all path configurations connecting the initial vertices to any permutation of the terminal vertices (other than the identity) have at least 
one intersection then

$$
Z\left(\mathbf{v}^{\mathbf{i}}, \mathbf{v}^{\mathbf{f}}\right)=\left|\begin{array}{cccc}
Z\left(v_{1}^{i}, v_{1}^{f}\right) & Z\left(v_{1}^{i}, v_{2}^{f}\right) & \ldots & Z\left(v_{1}^{i}, v_{n}^{f}\right) \\
Z\left(v_{2}^{i}, v_{1}^{f}\right) & Z\left(v_{2}^{i}, v_{2}^{f}\right) & \ldots & Z\left(v_{2}^{i}, v_{n}^{f}\right) \\
\cdot & \cdot & . & \cdot \\
\cdot & \cdot & . & \cdot \\
\cdot & \cdot & . & \cdot \\
Z\left(v_{n}^{i}, v_{1}^{f}\right) & Z\left(v_{n}^{i}, v_{2}^{f}\right) & \ldots & Z\left(v_{n}^{i}, v_{n}^{f}\right)
\end{array}\right|
$$

where $Z\left(v_{\alpha}^{i}, v_{\beta}^{f}\right)$ is the weighted sum over configurations of a single path starting at vertex $v_{\alpha}^{i}$ and ending at vertex $v_{\beta}^{f}$.

The theorem may be used to find the Hankel $n$-path contact polynomial by taking the point set of the half plane square lattice $\Xi$ to be the vertex set of the directed graph and the arc set to be the vertex pairs $(u, v)$ such that $v$ is either an up step or a down step away from vertex $u$. Weight $\kappa$ is assigned to the vertices with $y=0$ and weight one to all other vertices and all of the arcs. In this way each Hankel path configuration gets the correct weight $\kappa^{c}$ as in the defining equations (22) and (23). The elements of the determinant in this case are the single path contact polynomials.

\section{Corollary 1.}

$$
\hat{Z}_{2 r}^{H}(n ; \kappa)=\left|\begin{array}{llll}
\hat{Z}_{2 r}^{\mathcal{S}}(\kappa) & \hat{Z}_{2 r+2}^{\mathcal{S}}(\kappa) & \ldots & \hat{Z}_{2 r+2(n-1)}^{\mathcal{S}}(\kappa) \\
\hat{Z}_{2 r+2}^{\mathcal{S}}(\kappa) & \hat{Z}_{2 r+4}^{\mathcal{S}}(\kappa) & \ldots & \hat{Z}_{2 r+2 n}^{\mathcal{S}}(\kappa) \\
\hat{Z}_{2 r+4}^{\mathcal{S}}(\kappa) & \hat{Z}_{2 r+6}^{\mathcal{S}}(\kappa) & \ldots & \hat{Z}_{2 r+2(n+1)}^{\mathcal{S}}(\kappa) \\
\ldots & \ldots & \ldots & \ldots \\
\hat{Z}_{2 r+2(n-1)}^{\mathcal{S}}(\kappa) & \hat{Z}_{2 r+2 n}^{\mathcal{S}}(\kappa) & \ldots & \hat{Z}_{2 r+4(n-1)}^{\mathcal{S}}(\kappa)
\end{array}\right|
$$

Replacing $\kappa$ by $\kappa(\omega)$ in corollary 1 and using the definitions (6) and (20) gives

$$
\hat{H}_{2 r}^{(n)}(\omega)=\operatorname{det}\left(\hat{H}_{2(r+\alpha+\beta-2)}^{\mathcal{S}}(\omega)\right)_{1 \leq \alpha, \beta \leq n}
$$

Note, determinant (32) is a Hankel determinant as the matrix elements appearing in the determinant only depend on the sum of the row and column indexes, $\alpha$ and $\beta$.

Theorem 3. For any $k$ such that $0 \leq k \leq n-1$

$$
\hat{H}_{2 r}^{(n)}(\omega)=\operatorname{det}(M(k))
$$

where $M(k)$ is the matrix $\left(M_{\alpha \beta}(k)\right)_{1 \leq \alpha, \beta \leq n}$ with

$$
M_{\alpha \beta}(k)= \begin{cases}\hat{H}_{2(r+\alpha+\beta-2)}^{\mathcal{S}}(0)=C_{r+\alpha+\beta-2} & \text { for } \beta \leq k \\ \hat{H}_{2(r+\alpha+\beta-2)}^{\mathcal{S}}(\omega) & \text { for } \beta>k\end{cases}
$$


In particular when $k=n-1$

$$
\hat{H}_{2 r}^{(n)}(\omega)=\left|\begin{array}{lllll}
C_{r} & C_{r+1} & \ldots & C_{r+n-2} & \hat{H}_{2(r+n-1)}^{\mathcal{S}}(\omega) \\
C_{r+1} & C_{r+2} & \ldots & C_{r+n-1} & \hat{H}_{2(r+n)}^{\mathcal{S}}(\omega) \\
C_{r+2} & C_{r+3} & \ldots & C_{r+n} & \hat{H}_{2(r+n+1)}^{\mathcal{S}}(\omega) \\
\vdots & \vdots & \ldots & \vdots & \vdots \\
C_{r+n-1} & C_{r+n} & \ldots & C_{r+2 n-3} & \hat{H}_{2(r+2 n-2)}^{\mathcal{S}}(\omega)
\end{array}\right|
$$

Algebraic proof. We show that while $k<n-1$, increasing $k$ leaves the determinant unchanged and the result follows by induction since it is true for $k=0$.

From (10) we obtain the recurrence relation

$$
\hat{H}_{2 r}^{\mathcal{S}}(\omega)=C_{r}+\omega \hat{H}_{2 r+2}^{\mathcal{S}}(\omega)
$$

and applying this to column $k+1$ of determinant (32)

$$
\hat{H}_{2(r+\alpha+k-1)}^{\mathcal{S}}(\omega)=C_{r+\alpha+k-1}+\omega \hat{H}_{2(r+\alpha+k+1)}^{\mathcal{S}}(\omega)
$$

Hence $\operatorname{det}(M(k))$ is the sum of two determinants the first of which is $\operatorname{det}(M(k+1))$ and the second evaluates to zero since it has two equal columns $\left(\hat{H}_{2(r+\alpha+k+1)}^{\mathcal{S}}(\omega)\right.$ is column $k+2)$.

Combinatorial proof. Expanding out the determinant (32)

$$
\hat{H}_{2 r}^{(n)}(\omega)=\sum_{\boldsymbol{\sigma} \in P_{n}} \epsilon_{\boldsymbol{\sigma}} \prod_{\beta=1}^{n} \hat{H}_{2\left(r+\sigma_{\beta}+\beta-2\right)}^{\mathcal{S}}(\omega)
$$

where $P_{n}$ is the set of permutations of $\{1,2, \ldots, n\}, \epsilon_{\boldsymbol{\sigma}}$ is the sign of $\boldsymbol{\sigma}$ and $\sigma_{\beta}$ is the image of $\beta$ under the permutation $\boldsymbol{\sigma}$. Now from (14)

$$
\hat{H}_{2\left(r+\sigma_{\beta}+\beta-2\right)}^{\mathcal{S}}(\omega)=\sum_{b=0}^{\infty}\left|\{\stackrel{\text { 艺 }}{\Delta}\}_{2\left(r+\sigma_{\beta}+\beta-2\right), 2 b}\right| \omega^{b} .
$$

where the bi-ballot line $\mathcal{L}_{\beta}$ for the set of bi-ballot paths $\left\{\stackrel{M^{*}}{\Delta}\right\}_{2\left(r+\sigma_{\beta}+\beta-2\right), 2 b}$ passes through $(2(r+\beta-1), 0)$. Substituting in $(38)$ gives

$$
\left[\omega^{b}\right] \hat{H}_{2 r}^{(n)}(\omega)=\sum_{\boldsymbol{\sigma} \in P_{n}} \epsilon_{\boldsymbol{\sigma}} \sum_{\mathbf{b}_{n} \in \mathbb{K}_{n}^{b}} \prod_{\beta=1}^{n}\left|\left\{\stackrel{M^{*}}{\Delta}\right\}_{2\left(r+\sigma_{\beta}+\beta-2\right), 2 b_{\beta}}\right|
$$

where $\mathbb{K}_{n}^{b}$ is the set of compositions of $b$ into exactly $n$ parts i.e the set of all $n$-tuples, $\mathbf{b}_{n}=\left(b_{1}, b_{2}, \ldots, b_{n}\right), b_{\beta} \geq 0$, such that $\sum_{\beta=1}^{n} b_{\beta}=b$. Given $k \leq n-1$, we can partition $\mathbb{K}_{n}^{b}$ as $\mathbb{K}_{n}^{b}=\mathbb{K}_{n, k}^{b} \cup \overline{\mathbb{K}}_{n, k}^{b}$ where $\mathbb{K}_{n, k}^{b}$ is the set of compositions in which $b_{\beta}>0$ for at least one $\beta \in\{1,2, \ldots, k\}$ and $\overline{\mathbb{K}}_{n, k}^{b}=\mathbb{K}_{n}^{b}-\mathbb{K}_{n, k}^{b}$. Restricting the sum over compositions in (40) to $\overline{\mathbb{K}}_{n, k}^{b}$ is equivalent to setting $\omega=0$ in the first $k$ columns of the determinant, (32) 
which is then the determinant of $M_{\alpha \beta}(k)$. The theorem would therefore be proven if we could show that the sum over the set $\mathbb{K}_{n, k}^{b}$ is zero. This will be achieved by constructing a bijection which matches configurations corresponding to permutations of opposite parity resulting in pairwise cancellation of all terms associated with $\mathbb{K}_{n, k}^{b}$.

The sum restricted to $\mathbb{K}_{n, k}^{b}$ can be written

$$
\sum_{\boldsymbol{\sigma} \in P_{n}} \epsilon_{\boldsymbol{\sigma}} \sum_{\mathbf{b}_{n} \in \mathbb{K}_{n, k}^{b}} \prod_{\beta=1}^{n}\left|\{\stackrel{\text { 艺 }}{厶}\}_{2\left(r+\sigma_{\beta}+\beta-2\right), 2 b_{\beta}}\right|=\left|\Omega_{k}^{+}\right|-\left|\Omega_{k}^{-}\right|
$$

where, with $P_{n}^{ \pm}$the set of even/odd permutations of $\{1,2, \ldots, n\}$ and the $n$-path $\mathbf{T}=$ $\left\{T_{1}, T_{2}, \ldots, T_{n}\right\}$,

$$
\Omega_{k}^{ \pm}=\left\{(\boldsymbol{\sigma}, \mathbf{T}) \mid \boldsymbol{\sigma} \in P_{n}^{ \pm}, T_{\beta} \in\{\stackrel{\#}{M}\}_{2\left(r+\sigma_{\beta}+\beta-2\right), 2 b_{\beta}}, \mathbf{b}_{n} \in \mathbb{K}_{n, k}^{b}\right\} .
$$

A typical member of $\Omega_{2}^{-}$is shown in figure 10 . We now define the bijection $\Phi_{\omega}: \Omega_{k}^{+} \leftrightarrow \Omega_{k}^{-}$.

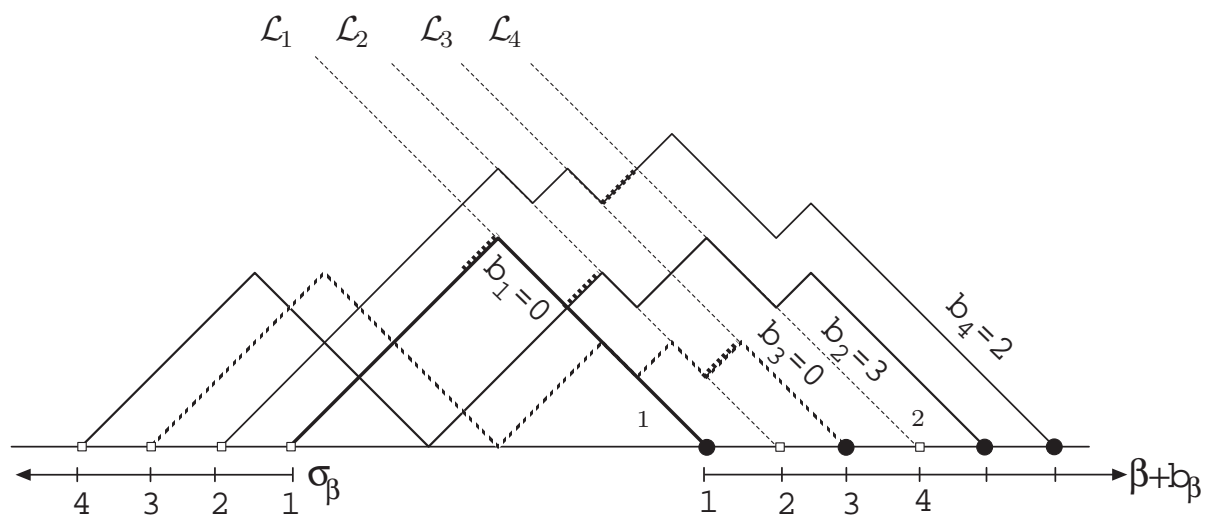

Figure 10: An example of the path configurations arising in the set $\Omega_{2}^{-}$, showing the path and terminal labelling for a given permutation, $\boldsymbol{\sigma}=1432$ and $r=6$.

Let $\Omega_{k}=\Omega_{k}^{+} \cup \Omega_{k}^{-}$. If $\Omega_{k}=\phi$ then the sum (41) is zero and it is sufficient to consider the case $\Omega_{k} \neq \phi$. In this case there exists $b_{\beta}, 1 \leq \beta \leq k$ such that $b_{\beta}>0$ which implies the existence of $\beta_{\min }=\min \left\{\beta \mid b_{\beta}>0\right\} \leq k<n$. Let $\sharp_{\beta_{\min }}$ be the bridge of $T_{\beta_{\min }}$ and since $\beta_{\text {min }}<n, T_{\beta_{\min }+1}$ exists with bridge $\sharp_{\beta_{\min }+1}$.

The image pair $\left(\boldsymbol{\sigma}^{\prime}, \mathbf{T}^{\prime}\right)=\Phi_{\omega}((\boldsymbol{\sigma}, \mathbf{T}))$ is defined by

- $T_{\beta}^{\prime}=T_{\beta}$, for $\beta \neq \beta_{\min }, \beta_{\min }+1$,

- $T_{\beta_{\min }}^{\prime}$ is the path $T_{\beta_{\min }+1}$ but with a bridge $\sharp_{\beta_{\min }+1}$ moved to the rightmost up step (which always exists since $\sharp_{\beta_{\min }+1}$ is to the right of $\sharp_{\beta_{\min }}$ ) of $T_{\beta_{\min }+1}$ to the left of $\sharp_{\beta_{\text {min }}+1}$ - e.g. see figure 11, 
- $T_{\beta_{\min }+1}^{\prime}$ is the path $T_{\beta_{\min }}$ but with a bridge $\sharp_{\beta_{\min }}$ moved to the leftmost up step (which always exists as $b_{\beta_{\min }}>0$ ) of $T_{\beta_{\min }}$ to the right of $\sharp_{\beta_{\min }}$,

- $\sigma_{\beta}^{\prime}=\sigma_{\beta}, \beta \neq \beta_{\min }, \beta_{\min }+1, \sigma_{\beta_{\min }}^{\prime}=\sigma_{\beta_{\min }+1}$ and $\sigma_{\beta_{\min }+1}^{\prime}=\sigma_{\beta_{\min }}$ Note, this is a transposition of $\boldsymbol{\sigma}$ hence $\epsilon_{\boldsymbol{\sigma}^{\prime}}=-\epsilon_{\boldsymbol{\sigma}}$.

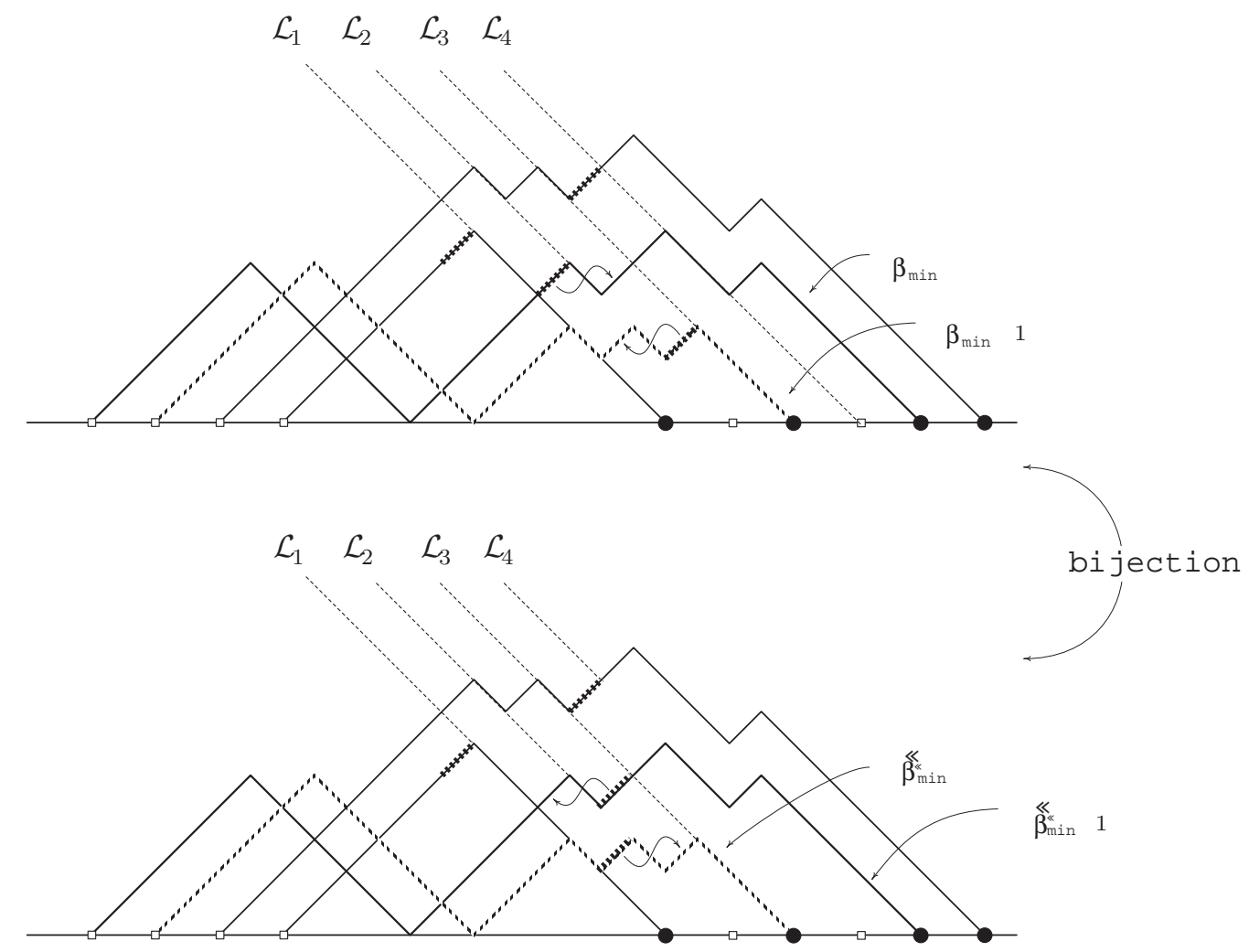

Figure 11: An example of the bijection $\Phi_{\omega}$.

Moving the bridge on $T_{\beta_{\min }}$ to the right decreases $b_{\beta_{\min }}$ by one and moving the bridge on $T_{\beta_{\min }+1}$ to the left increases $b_{\beta_{\min }+1}$ by one and hence $b_{\beta_{\min }}^{\prime}=b_{\beta_{\min }+1}+1$ and $b_{\beta_{\min }+1}^{\prime}=$ $b_{\beta_{\min }}-1$. The remaining $b$ 's are unchanged, thus $b_{\beta}^{\prime}=b_{\beta}, \beta \neq \beta_{\min }, \beta_{\min }+1$. Hence the value of $b$ is unchanged. Since $T_{\beta_{\min }}^{\prime}$ terminates at $v_{\beta_{\min }+1}^{f}, T_{\beta_{\min }+1}^{\prime}$ terminates at $v_{\beta_{\min }}^{f}$ and $b_{\beta_{\min }}^{\prime}>0$ we have $\left(\boldsymbol{\sigma}^{\prime}, \mathbf{T}^{\prime}\right) \in \Omega_{k}$. Furthermore, since $\epsilon_{\boldsymbol{\sigma}^{\prime}}=-\epsilon_{\boldsymbol{\sigma}}$, if $(\boldsymbol{\sigma}, \mathbf{T}) \in \Omega_{k}^{ \pm}$, then $\left(\boldsymbol{\sigma}^{\prime}, \mathbf{T}^{\prime}\right) \in \Omega_{k}^{\mp}$.

Is $\Phi_{\omega}^{2}=1$ ? Let $\left(\boldsymbol{\sigma}^{\prime \prime}, \mathbf{T}^{\prime \prime}\right)=\Phi_{\omega}\left(\left(\boldsymbol{\sigma}^{\prime}, \mathbf{T}^{\prime}\right)\right)$. Since $b_{\beta}^{\prime}=b_{\beta}=0$ for $\beta<\beta_{\min }$ and $b_{\beta_{\min }}^{\prime}=b_{\beta_{\min }+1}+1>0$ we have $\beta_{\min }^{\prime}=\beta_{\min }$. Moving the bridge $\sharp_{\beta_{\min }^{\prime}}$ forward places it on the edge previously labeled $\sharp_{\beta_{\min }+1}$ and moving $\sharp_{\beta_{\min }^{\prime}+1}$ back places it on the edge previously labeled $\sharp_{\beta_{\min }}$ and hence $T_{\beta_{\min }+1}^{\prime \prime}=T_{\beta_{\min }+1}$ and $T_{\beta_{\min }}^{\prime \prime \prime}=T_{\beta_{\min }}$. Furthermore, since $\beta_{\min }^{\prime}=\beta_{\min }$ we have $\boldsymbol{\sigma}^{\prime \prime}=\boldsymbol{\sigma}$. Thus $\left(\boldsymbol{\sigma}^{\prime \prime}, \mathbf{T}^{\prime \prime}\right)=(\boldsymbol{\sigma}, \mathbf{T})$ and hence $\Phi_{\omega}$ is a bijection. Hence $\left|\Omega_{k}^{+}\right|=\left|\Omega_{k}^{-}\right|$and the sum in (41) is zero. 


\subsection{Product form for the number of Ceratic Hankel $n$-path con- tact polynomials.}

Substitution of (10) in (35) enables the coefficients in the $\omega$ expansion of the Hankel $n-$ path contact polynomial (20) to be expressed as a determinant involving only Catalan numbers.

$$
\hat{h}_{r}^{(n)}(b)=\left|\begin{array}{lllll}
C_{r} & C_{r+1} & \ldots & C_{r+n-2} & C_{r+n-1+b} \\
C_{r+1} & C_{r+2} & \ldots & C_{r+n-1} & C_{r+n+b} \\
C_{r+2} & C_{r+3} & \ldots & C_{r+n} & C_{r+n+1+b} \\
& & & & \\
\ldots & \ldots & \ldots & \ldots & \ldots \\
C_{r+n-1} & C_{r+n} & \ldots & C_{r+2 n-3} & C_{r+2 n-2+b}
\end{array}\right| .
$$

Since $C_{r}$ is the number of Dyck paths of length $2 r$, Theorem 2 shows that $\hat{h}_{r}^{(n)}(b)$ can be interpreted as the number of unweighted Hankel $n$-paths in which the terminal vertex of the $n^{\text {th }}$ path is shifted through a distance $2 b$ to $(2(r+n-1+b), 0)$. These paths are what we have called Ceratic Hankel paths.

For $n=1$

$$
\hat{h}_{r}^{(1)}(b)=C_{r+b}
$$

in agreement with (10), and evaluation of the determinant for $n=2$ gives

$$
\hat{h}_{r}^{(2)}(b)=\frac{6(b+1)}{(r+2)(r+b+3)} C_{r} C_{r+b+1} .
$$

For $n \geq 3, \hat{h}_{r}^{(n)}(b)$ can be obtained using the following recurrence relation which follows by applying Dodgson's formula [13] to the determinant.

$$
\hat{h}_{r}^{(n)}(b)=\left[\hat{h}_{r}^{(n-1)}(0) \hat{h}_{r+2}^{(n-1)}(b)-\hat{h}_{r+1}^{(n-1)}(0) \hat{h}_{r+1}^{(n-1)}(b)\right] / \hat{h}_{r+2}^{(n-2)}(0) .
$$

This relation can be used to verify the following explicit formula.

Theorem 4. The coefficients in the $\omega$ expansion (20) of the Hankel $n$-path contact polynomial can be expressed in the product form

$$
\hat{h}_{r}^{(n)}(b)=\hat{h}_{r}^{(n)}(0) f_{r}^{(n)}(b)
$$

where

$$
\hat{h}_{r}^{(n)}(0)=\prod_{i=1}^{n} \frac{(2 r+2 i-2) !(2 i-1) !}{(r+i-1) !(r+i+n-1) !}
$$

and

$$
f_{r}^{(n)}(b)=\left(\begin{array}{c}
n+b-1 \\
b
\end{array}\right) \frac{(2 r+2 n-1)_{2 b}}{(r+n)_{b}(r+2 n)_{b}}
$$

Proof. The formula agrees with (44) and (45) for $n=1$ and 2 and lengthy manipulation shows that it also satisfies the recurrence relation (46). 


\subsection{Recurrence relations for Ceratic Hankel paths}

From (46) using the definition (20), or applying Dodgson's formula to (35) gives

$$
\hat{H}_{2 r}^{(n)}(\omega)=\left[\hat{H}_{2 r}^{(n-1)}(0) \hat{H}_{2 r+4}^{(n-1)}(\omega)-\hat{H}_{2 r+2}^{(n-1)}(0) \hat{H}_{2 r+2}^{(n-1)}(\omega)\right] / \hat{H}_{2 r+4}^{(n-2)}(0)
$$

where $\hat{H}_{r}^{(n)}(0)=\hat{h}_{r}^{(n)}(0)$ is given by $(48)$.

The following recurrence relation, which is first order in both $n$ and $r$ follows from Theorem 4 after lengthy manipulation.

$$
\hat{h}_{r-1}^{(n-1)}(0) \hat{h}_{r}^{(n)}(b-1)=\hat{h}_{r}^{(n-1)}(0) \hat{h}_{r-1}^{(n)}(b)-\hat{h}_{r-1}^{(n)}(0) \hat{h}_{r}^{(n-1)}(b) .
$$

which implies

$$
\omega \hat{H}_{2 r-2}^{(n-1)}(0) \hat{H}_{2 r}^{(n)}(\omega)=\hat{H}_{2 r}^{(n-1)}(0) \hat{H}_{2 r-2}^{(n)}(\omega)-\hat{H}_{2 r-2}^{(n)}(0) \hat{H}_{2 r}^{(n-1)}(\omega) .
$$

In [5] it was shown, in the context of $\hat{Z}_{2 r}^{\mathrm{W}}(n ; \kappa)$, that combining this relation with (50) gives the second order recurrence relation for fixed $n$

$$
\omega \hat{H}_{2 r+4}^{(n)}(\omega)-\left(\omega \rho_{r}^{(n)}+\rho_{r+1}^{(n-1)}\right) \hat{H}_{2 r+2}^{(n)}(\omega)+\rho_{r-1}^{(n)} \rho_{r+1}^{(n-1)}\left(1+\frac{\mu_{r-1}^{(n)}}{\mu_{r}^{(n-1)}}\right) \hat{H}_{2 r}^{(n)}(\omega)=0
$$

where

$$
\rho_{r}^{(n)}=\frac{\hat{h}_{r+1}^{(n)}(0)}{\hat{h}_{r}^{(n)}(0)}=\frac{(2 r+1)_{2 n}}{(r+1)_{2 n}}
$$

and

$$
\mu_{r}^{(n)}=\frac{\hat{h}_{r+1}^{(n+1)}(0)}{\hat{h}_{r+1}^{(n)}(0)}=\frac{(2 n+1) !(2 r) !}{r !(2 n+r+1) !}=\frac{(r+1)_{r}}{(2 n+2)_{r}} .
$$

This leads to the explicit form of the recurrence relation

$$
\begin{aligned}
& \omega(r+2 n-2)(r+n-1)_{n-1}^{2} \hat{H}_{2 r}^{(n)}(\omega) \\
& -4^{n-1}\left(r-\frac{1}{2}\right)_{n-1}(r+n-1)_{n-1}(2(2 r-3) \omega+r+2 n-2) \hat{H}_{2 r-2}^{(n)}(\omega) \\
& \quad+16^{n-1} 2(2 r-3)\left(r-\frac{1}{2}\right)_{n-1}^{2} \hat{H}_{2 r-4}^{(n)}(\omega)=0
\end{aligned}
$$

\section{Acknowledgements}

Financial support from the Australian Research Council is gratefully acknowledged. JWE is also grateful for the kind hospitality provided by the University of Melbourne and RB is also grateful for the kind hospitality provided by Royal Holloway College, London University. 


\section{References}

[1] R. Brak, J. W. Essam, and A. Owczarek. New results for directed vesicles and chains near an attractive wall. J. Stat. Phys., 93:155-192, 1998.

[2] R. Brak, J. Essam, and A. L. Owczarek. Exact solution of $n$ directed non-intersecting walks interacting with one or two boundaries. J. Phys. A., 32:2921-2929, 1999.

[3] R. Brak, J. Essam, and A. L. Owczarek. Partial difference equation method for lattice path problems. Annals of Comb., 3:265-275, 1999.

[4] R. Brak and J. W. Essam. Directed percolation near a wall: III. excact results for the mean length and number of contacts. J. Phys. A., 31:4851-4869, 1999.

[5] R. Brak, J. Essam, and A. L. Owczarek. Scaling analysis for the absorption transition in a watermelon network of $n$ directed non-intersecting walks. J. Phys. A., 102:997, 2001.

[6] R. Brak, J. Essam, and A. L. Owczarek. From the Bethe Ansatz to the Gessel-Viennot theorem. Annals of Comb., 3:251-263, 1998.

[7] R. Brak and J. Essam. Return polynomials for non-intersecting paths above a surface on the directed square lattice. Submitted to J. Phys. A. 2001.

[8] H. Gould. Some generalizations of Vandermond'e convolution. Amer. Mathematics Monthly., 63:84-91. 1956.

[9] M. E. Fisher. J. Stat. Phys., 34:667, 1984.

[10] X. Viennot. A combinatorial interpretation of the quotient-difference algorithm. Preprint, 1986.

[11] I. Gessel and X. Viennot. Binomial determinants, paths, and hook length formulae. Adv. Math., 58:300-321, 1985.

[12] I. Gessel and X. Viennot. Determinants, paths, and plane paritions. 1985. Preprint.

[13] C. L. Dodgson. Proc. Roy. Soc. London, 1866. 